\title{
Modeling and Analyzing Anycast and Geocast Routing in Wireless Mesh Networks
}

\author{
Fazle Hadi*, Sheeraz Ahmed ${ }^{\dagger}$, Abid Ali Minhas ${ }^{\ddagger}$, Atif Naseer ${ }^{\S}$ \\ *Preston University Kohat, Peshawar Campus, Pakistan \\ ${ }^{\dagger}$ Preston University Kohat, Peshawar Campus, Pakistan \\ $\ddagger$ Al Yamamah University Riyadh, Saudi Arabia \\ Science and Technology Unit, Umm Al Qura University, Saudi Arabia
}

\begin{abstract}
Wireless technology has become an essential part of this era's human life and has the capability of connecting virtually to any place within the universe. A mesh network is a self healing wireless network, built through a number of distributed and redundant nodes to support variety of applications and provide reliability. Similarly, anycasting is an important service that might be used for a variety of applications. In this paper we have studied anycast routing in the wireless mesh networks and the anycast traffic from the gateway to the mesh network having multiple anycast groups. We have also studied the geocast traffic in which the packets reach to the group head via unicast traffic and then are broadcasted inside the group. Moreover, we have studied the intergroup communication between different anycast groups. The review of the related literature shows that no one has considered anycasting and geocasting from gateway to the mesh network while considering the multiple anycast groups and intergroup communication. The network is modeled, simulated and analyzed for its various parameters using OMNET++ simulator.
\end{abstract}

Keywords-Mesh Network; Anycast; Geocast; Routing; Unicast

\section{INTRODUCTION}

The basic aim of the wireless mesh networks (WMNs) is to guarantee the connectivity. WMNs are gaining popularity for its wide range of applications. The networks have gained substantial consideration as an unconventional solution to applications such as community networks, enterprise networks, and last mile access networks to the Internet [1]. WMNs are citywide multi-hop networks. They have a fixed infrastructure in the form of gateways and either mobile or fixed wireless mesh clients. The gateways have neither the mobility nor the power issues. There might be a series of fixed points- they relay the traffic to the sparsely distributed nodes. Gateways provide the internet access to the wireless nodes. This is the most common application of the wireless mesh networks. Video on demand and IP-TV are other interesting applications of a high speed wireless mesh networks. Among all these applications group communication is an important paradigm to study. Considering the citywide mesh network there might be many groups like the group of educational institutes, industries, vehicular network clusters etc. Contemporary studies mostly focus on optimal reaching to a gateway for the internet access.

Routing in the wireless mesh networks always attracts the researchers. Field base routing (FBR) is recently introduced for WMNs [2]. FBR relies on a routing field, and it is exchanged among the participating nodes. The data travels along the path having a relatively larger value of heat (the value computed

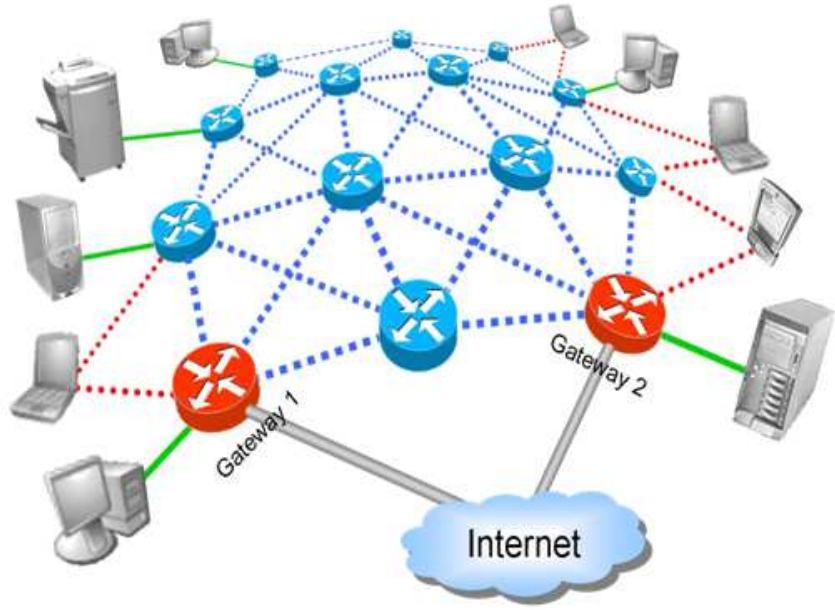

Fig. 1. Basic mesh architecture [21]

for every node considering the gateway as a source of heat) [2].

Anycast is an important service that always applies the greedy approach to deliver the packets to the nearest destination. If there are various groups of the same category then the anycast traffic will be forwarded to the next hop towards the group head having larger calculated parameter (temperature field). It considers the group head as the heat source.

The major contribution of this study is the proposal of an anycast model for the traffic from gateway to the mesh nodes using various anycast groups. Moreover, the study also analyzed the geocast and unicast communication. To the best of our knowledge the anycast and geocast communication have not been studied for the traffic forwarded by mesh gateways to the mesh clients. Though in [3] Tracy Camp et al. studied Geocast Adaptive Mesh Environment for Routing (GAMER) and the presented technique is about the geocast communication in ad hoc network. But the geocast technique presented in this paper is for WMNs and specifically for the traffic generated by gateway to mesh clients based on the field base routing. The geocasting is achieved by delivering the packets using unicast to a group head and then is broadcasted within the group. In addition to geocasting the main contribution of this study is the presentation of anycast model and group communication.

The existing literature does not handle multiple groups. So the inter-group communication is not yet covered. These issues 
are of serious apprehension, thats why it is the main focus of this study. The research work will also consider multiple anycast groups, in the result the inter-group communication becomes possible.

The rest of this paper is organized as follows. Related

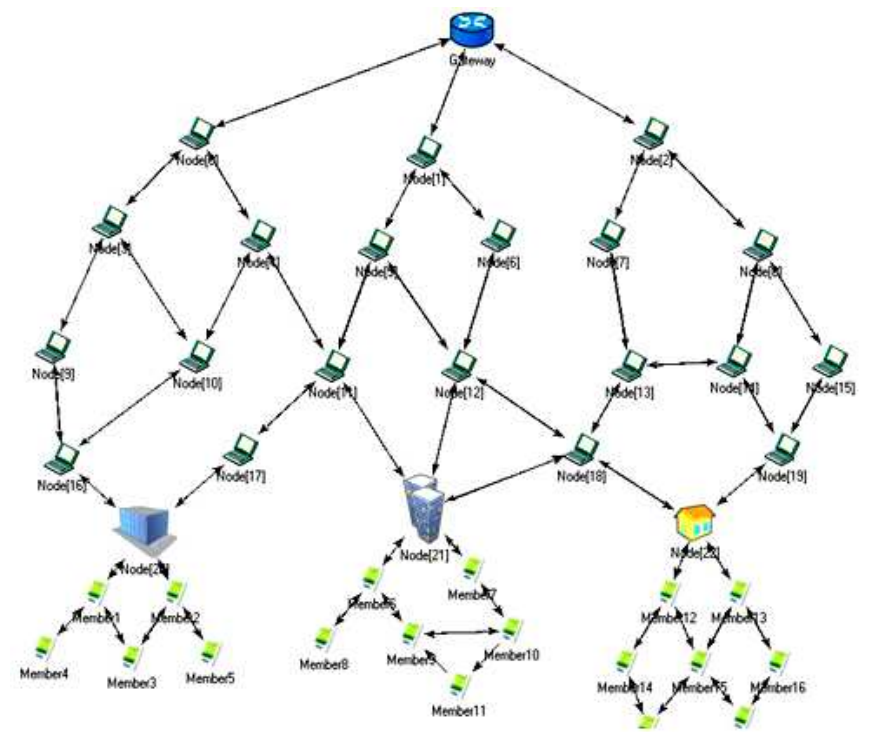

Fig. 2. Architecture of mesh network for anycast routing

work is underscored in section II. Section III describes the various challenges encountered in the design and analysis of wireless network structures like WMNs. Section IV depicts our proposed model for anycasting in WMNs, and the section $\mathrm{V}$ spells out the simulation environment and discussion on various traffic results. Finally, section VI concludes the paper with future directions.

\section{RELATED WORK}

Due to its demanding structure and application oriented architecture, there is a rich literature available about the wireless mesh networks. But after discussing the basic architecture we will converge our attention to the routing and group communication in the WMNs.

Akyildiz et al. [1] states that the wireless mesh network is a class of network where some nodes are fixed. These nodes serve as the gateway for the Internet connectivity. Others nodes are mobile, give access to the mobile nodes in a multihop fashion. Due to the redundant links, connectivity is not an issue in these networks. Most of the recent studies regarding the routing in the wireless mesh networks focus on the traffic flows from mesh nodes to the mesh gateways, for example, AODV [4] or OLSR [5]. Various unicast routing algorithms have been proposed in different studies like in [4], [5], [6] and [7]. Because of its scalability and robustness many researchers like Lenders et al. [8] and V. Park et al. [9] presented the field based routing algorithms. Recently, Baumann et al. [2] presented a field based routing algorithm for routing the packets from the mesh nodes to the gateway in anycast fashion. The authors give a field based routing algorithm, HEAT, it computes the temperature field keeping the gateway as the source of heat. In their later work Baumann et al. [10] presented the gateway source routing (GSR) algorithm for routing the packets through the wireless mesh network. The authors use the routing path in backward direction, the path which is build up by the mesh clients by sending the packets to the gateway. In order to route the packets from gateway to the mesh nodes it is necessary that the mesh clients first send the data to the gateway. it seems to be very anomalous limitation of the proposed scheme.

The concept of the field based routing algorithms is very straight forward. In these algorithms the data moves along the steepest path towards its destination. In [11], Bahr introduced a hybrid wireless mesh protocol (HWMP). It is the merging of two seemingly opposite technologies i.e. flexibility of ondemand route discovery and enabling efficient proactive routing as well.

Field base routing algorithms are ingeniously used for various applications like load balancing in wide area networks [12], data gathering in sensor networks [13], placement of sensor nodes [14] and routing in MANETs [2], [9]. This study also using the gradient based routing algorithm for routing unicast, anycast and geocast traffic in wireless mesh networks. We are presenting the anycast model considering the traffic from the gateway to the mesh clients, having different anycast groups. Geocasting is phenomenon in which packets are delivered to a particular group belonging to a specific geographical location. There are various geocast algorithms available in the literature e.g. [15] and [16] exclusively depends upon the exact geographical information of the source and the destination.

The exact geographical information need specialized devices and is very hard to obtain [17]. The authors also present a geocast model in which the traffic moves from the gateway till the group head in unicast fashion following the gradient base routing and then group head broadcasts it inside the group. In [18], the authors propose a joint traffic splitting, routing, rate control and scheduling algorithm called CLC-DGS, which splits and distributes network traffic into multiple gateways in an optimal way. The authors prove by extensive simulations that CLC-DGS can achieve maximum network utility and improves the performance of WMNs under various network environments including link and gateway heterogeneities and various interference models.

In paper [19], the authors propose a jamming technique which targets the periodic nature of the routing protocol residing in the network layer. The technique is based on the concept of null-frequency jamming which refers to periodic attacks targeting specific protocol period/frequency of operation. The effects of this jamming technique are investigated in stack, half-diamond, full-diamond, full-mesh and random topologies employing the optimised link state routing protocol. In order to fully utilize spectrum resource in WMNs, [20] proposes a combination of some communication techniques, including link scheduling, spatial reuse, power and rate adaptation and Network Coding (NC). This was done to activate as many transmission links as possible during one scheduling period, so that the total scheduling length can be minimized and network throughput can be maximized. They consider interplay among these techniques and present an optimal NC-aware link scheduling mechanism in multi-rate WMNs, which relies on the enumeration of all possible schedules. Due to the high computational complexity of proposed model, they utilize a column generation (CG)-based method to resolve the optimization problem. Also, they present a distributed power control algorithm, by which the computational complexity of the CG- 
based scheme can be largely reduced.

\section{ROUTING CHALLENGES IN WMNS}

There are various design and data transfer challenges in WMNs because of their applications and network topologies. Also, these challenges play a vital role in the design of routing algorithms for these networks as well the performances. Following factors are very important to be considered in the design of routing algorithms and analysis for WMNs.

1. Mobility of the nodes:

All the nodes represent an autonomous route for peer-to-peer connection.

2. Network topology:

A non-systematic mobility of nodes with varying speeds make the topology of the network vary randomly.

3. Link between the nodes:

The network nodes are connected by an air medium and do not have any fixed infrastructure.

4. Battery lifetime:

To retain the residual power in the nodes is a major trouble; so WMNs rely on batteries.

5. Network attacks:

Wireless networks have more chances of security attacks comparative to wired networks.

6. Quality of Service $(Q o S)$ :

The QoS depends on various parameters in delivery of data, resulting in lower performance.

7. Consumption of power:

The energy conservation plays an important part in network evaluation.

8. Bandwidth constraint:

The unfixed infrastructure based network has less throughput metrics than the fixed ones.

\section{Network Model}

In contrast to ad hoc networks, WMNs do not impose the strict infrastructure less property. The basis architecture of the wireless mesh network is shown in figure 1 [19]. It has some fixed nodes (gateways) which provide access to the Internet acting as the backbone. The other nodes may be fixed access points, provide connectivity to the wireless mobile nodes in multihop fashion. Every node in the network acts as a router.

In this paper, we have developed a scenario for the anycast routing in wireless mesh networks. Figure 2, shows the architecture of wireless mesh network for anycast routing. There are three types of nodes in this architecture.

1) Gateway: The gateway provides the Internet connectivity to the mesh clients. As in this paper we are considering the traffic from the gateway to the mesh clients so the gateway is the main source of traffic in this scenario.

2) Routing Nodes: These nodes have the capability to route the packet towards its destinations. The routing is based upon the Gradient-Based Routing. Routing field is calculated while keeping the destination as the ultimate source (maximum field value).

3) Groups: These are different groups having their own group members. Group members are registered with the group head. The group heads are registered with the gateway.

Presence of either anycast traffic at the gateway will be

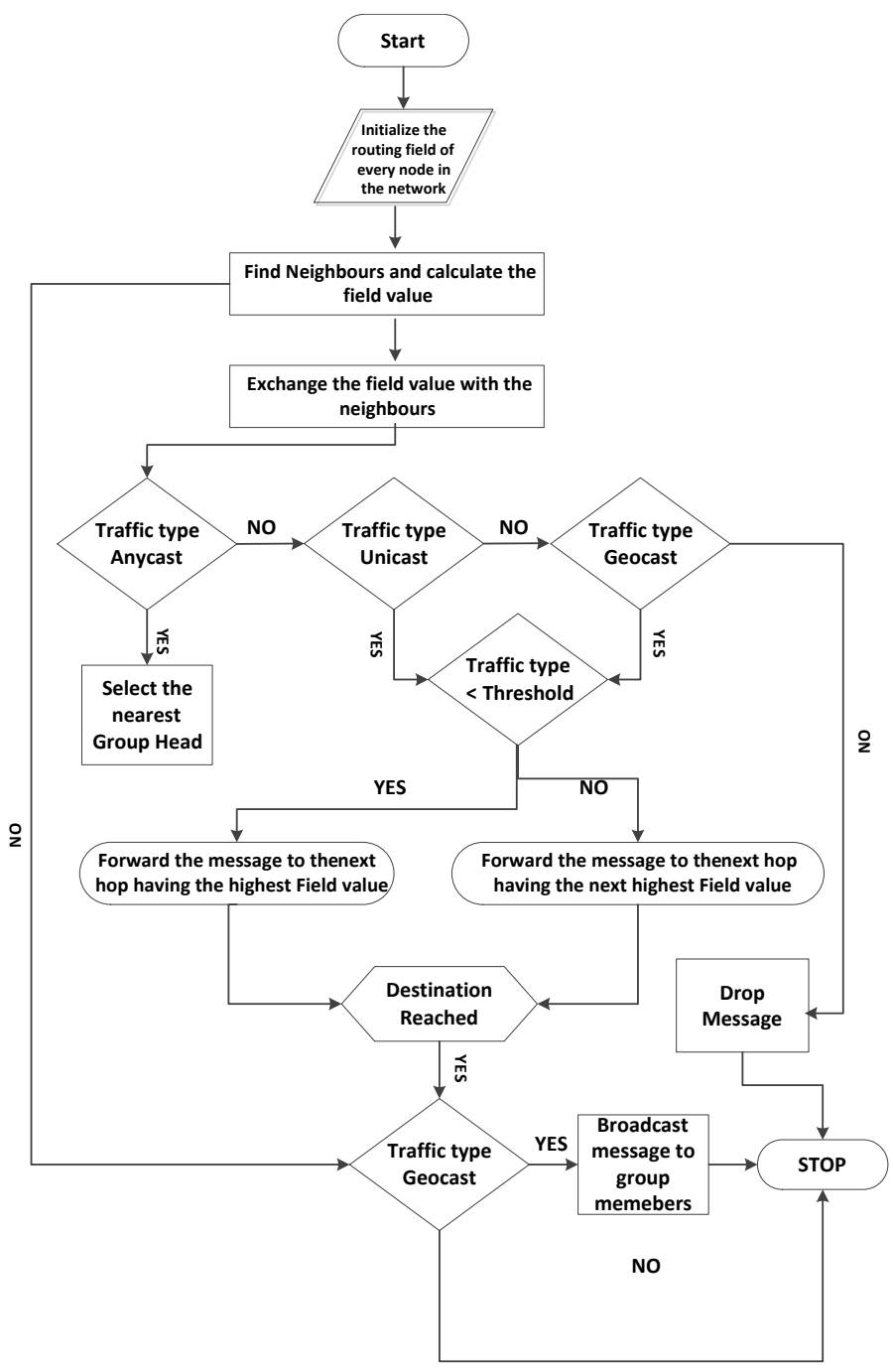

Fig. 3. Flow chart of WMN proposed routing

delivered to nearest group head via gradient based routing mechanism. If, on other hand, there is geocast traffic for any group, it will be routed to the group head following the gradient based routing in unicast fashion. It will then be broadcasted inside the group. Any routing node or group head may be regarded for the unicast traffic trending the same routing mechanism. There can be different interest groups suggested for various types of traffic. In such a way, intergroup communication is also made possible.

The routing field value is computed in the following equation. Let $y_{1}, \ldots . . y_{m}$ be the link paths of nodes in the network from their respective destinations and $d$ is the total distance of every node to its destination. Then:

$$
W_{i}=f\left(d_{i}\right)=\sum_{i=1}^{m}\left|y_{i}\right|=d
$$


which reflects the field value $f v$ of each sensor. Flow-chart in figure 3 depicts anycast, unicast and geocast routing with load balancing.

\section{A. Load Balancing}

Forwarding nodes make their decision on the basis of $f v$ of their neighbors. If proper load balancing technique is not followed then the entire traffic will follow the same route. This may not suit to energy constrained nodes. In our scheme, the load balancing is ensured by putting a threshold on the traffic flow. As traffic exceeds that of threshold value, the forwarding algorithm selects the next better route as shown in figure 3 .

\section{B. Algorithm}

The algorithm for anycast, unicast and geocast routing trending the gradient base routing technique with load balancing is explained below. Every sensor of the network computes its $f v$ considering the $f v$ of its neighbors and then advertising the value to update routing table of the neighbors. The packet is relayed to the next hop with the highest $f v$. This process continues till the traffic flow exceeds the threshold considered for load balancing. When the traffic flow exceeds the threshold, our algorithm selects next hop with the next highest $f v$. For geocast traffic it carries the data in unicast fashion till the group head and then broadcasts it inside the group. The detailed algorithm is given below:

\section{Gm: Group member \\ $T t$ : Traffic type \\ $F v$ : Field value \\ $N n$ : Neighbour node \\ $T h$ : Threshold value \\ $N h$ : Nearest head}

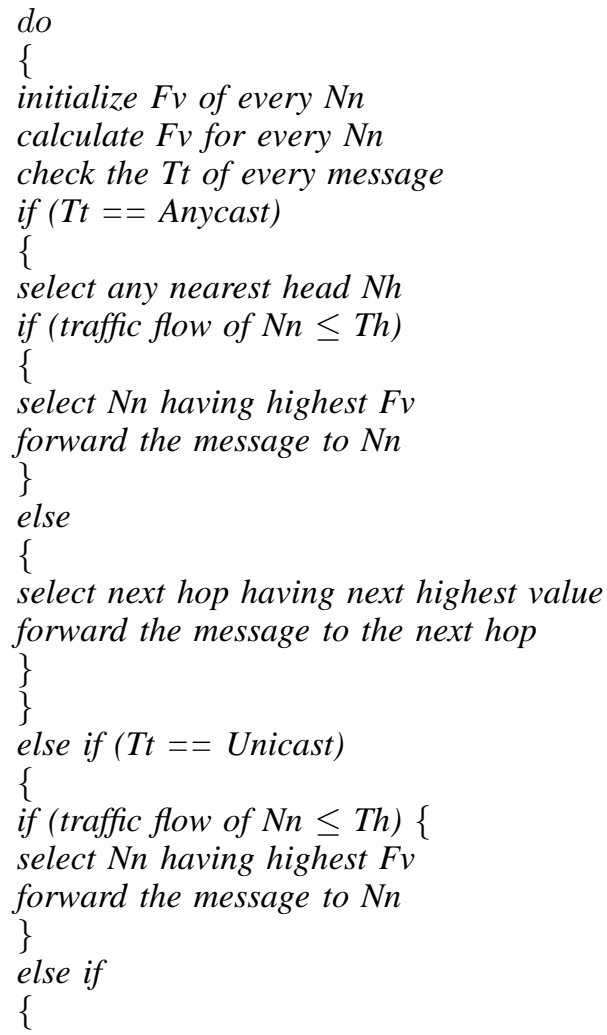

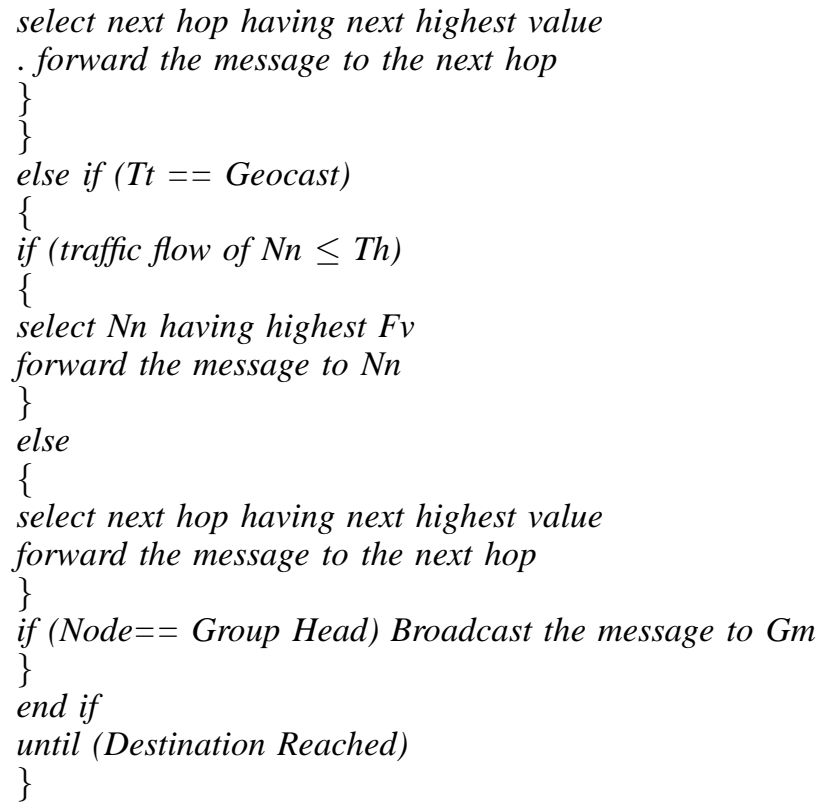

\section{Simulation Results}

For the performance analysis we have used OMNet++ [22] simulator. The scenario developed to simulate the anycast, unicast and geocast traffic contains twenty four nodes. It includes one gateway that relays the data from and to the Internet. The group heads recognize three anycast groups in this research, as we study the traffic from the Internet towards the mesh clients. The gateway is the ultimate source of the traffic. It relays the Internet traffic towards the mesh clients and groups.

Figure No. 4 depicts the comparison of packet delays

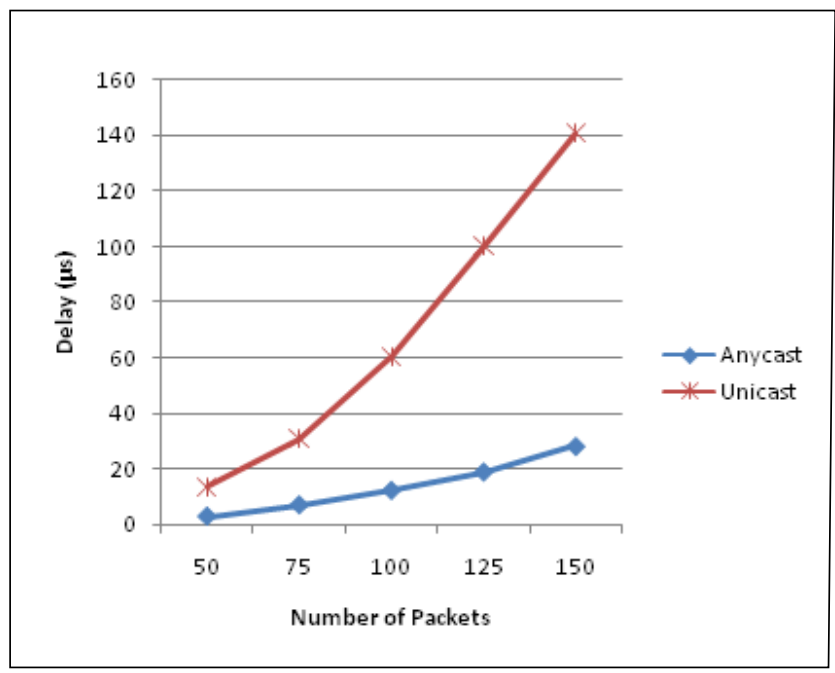

Fig. 4. Packet Delay comparison of Anycast and Unicast traffic

experienced by unicast and anycast traffic. As the idea of anycast group communication is proposed for the first time by considering the traffic from the gateway towards the mesh clients. We analyze the delay experienced by unicast and anycast traffic. Before the idea of anycast the only type of 
traffic flow was unicast. The analyses show that when the volume of data increases the delay experienced by unicast traffic is considerably increased as compared to the anycast traffic. This is because the anycast traffic always chooses the best possible path and delivers the packet to the nearest group head. As a precaution we have implemented the load balancing mechanism that diverts some traffic. The idea has been already explained earlier.

The packet delivery ratio is always an important parameter

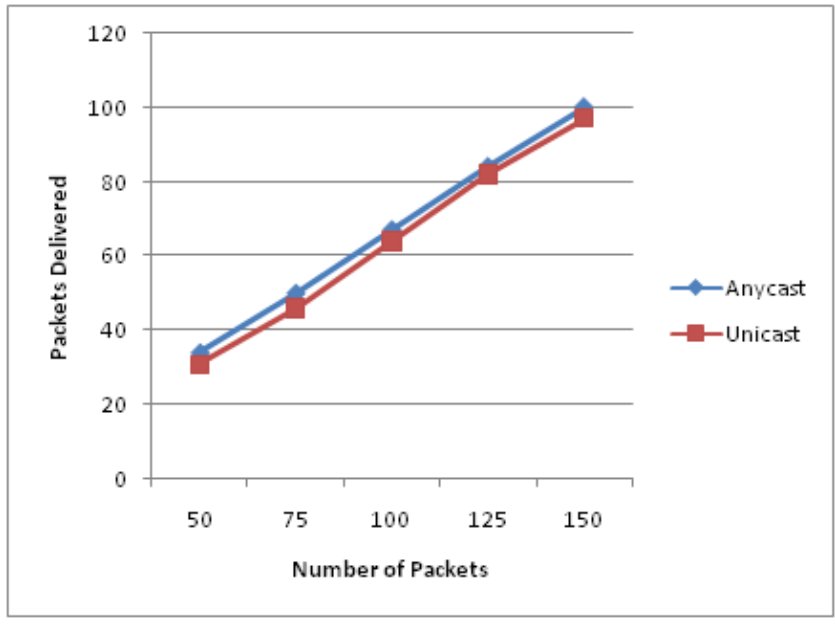

Fig. 5. Packet Delivery Ratio comparison of Anycast and Unicast traffic

to study. Figure 5 shows the comparison of the packet delivery ratio between the unicast and anycast type of traffic. Packets may be dropped uniformly in routing due to buffer overflow at the intermediate devices, link failure or any unseen problem. It has been observed that anycast type of traffic always gives better packet delivery ratio as compared to the unicast traffic. This is because that the unicast traffic will have to follow a predefine path towards a predefine destination and the anycast communication is adaptable and selects the best choice while forwarding the packets to the next hop towards any optimal destination.

Geocasting is the phenomenon of delivering the data packets to a particular geographical location. We consider the entire group members to be the part of a particular geographical location. Now, one way is to deliver the data packets to all group members in unicast fashion. We name it unicast based geocasting (UG). The other way is to deliver a packet to the group head (possible in the proposed group communication) and then broadcasts it within the group. We name it anycast based geocasting (AG). The figure 6 portrays the packet delay analysis experienced by $U G$ and AG. The traffic delay in case of UG is directly proportional to the number of group members and in case of AG it is a delay to reach to the group head plus the delay involved in the broadcast phase. The intra-group broadcasting delay has been considered in AG. Group members are normally in the direct range of the group head.

\section{CONCLUSION}

In this paper we have studied the anycast and geocast routing in wireless mesh networks. The concept of the

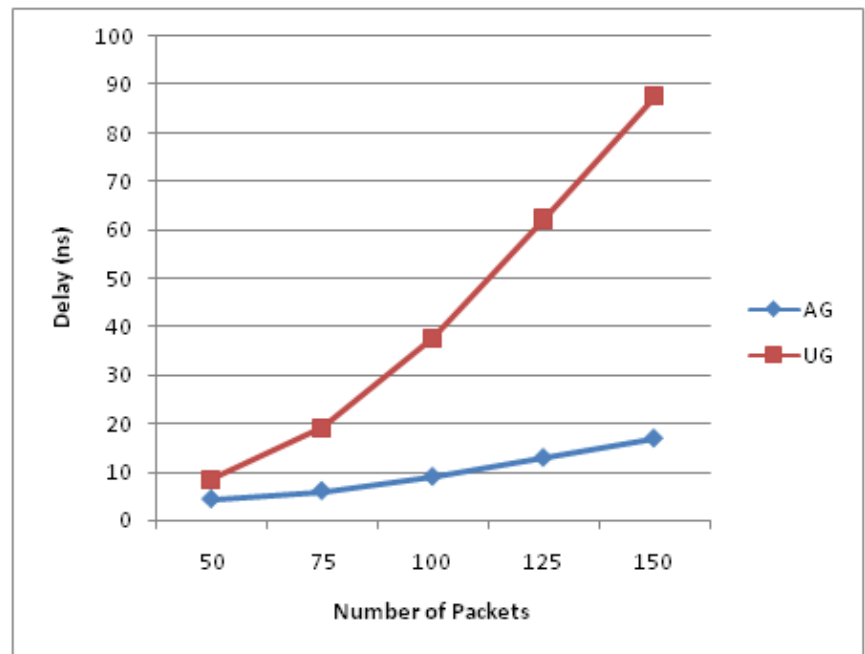

Fig. 6. Packet Delay comparison of Anycast and Unicast base Geocasting

anycast routing by considering the traffic from gateway towards the mesh clients has been proposed. The proposed technique has been studied against the unicast traffic by varying the volume of traffic from gateway towards the anycast groups. The anycast communication outperforms other type of communication in terms of packet delays and packet delivery ratio. Intergroup communication has also been made possible. Moreover geocast communication technique has been proposed in which the data travels in unicast fashion till the group head and then broadcasted inside the group. It lessens the delay time experienced by the geocasting based upon unicasting or geographical location. In future work we will create more realistic scenarios that will contain mobile nodes and adaptive group formation and head selection.

\section{REFERENCES}

[1] F. Akyildiz, X. Wang, and W. Wang, "Wireless mesh networks: a survey," Computer Networks, vol. 47, pp. 445-487, 15 March 2005.

[2] Rainer Baumann and Vincent Lenders and Simon Heimlicher and Martin May, "HEAT: Scalable Routing in Wireless Mesh Networks using Temperature Fields," in Proceedings of the IEEE WoWMoM,2007.

[3] Tracy Camp, Yu Liu, "An adaptive mesh-based protocol for geocast routing," Journal of Parallel and Distributed Computing archive Vol.63, No.2, 2003.

[4] Sung-Ju Lee, Elizabeth M. Belding-Royer, Charles E. Perkins, "Scalability study of the ad hoc on-demand distance vector routing protocol," International Journal of Network Management, vol. 13, No. 2, pp.97-114, 2003.

[5] T. Clausen and P. Jacquet, "Optimized Link State Routing Protocol," IETF Internet Draft, draft-ietf-manet-olsr-11.txt, July 2003.

[6] D. B. Johnson and D. A. Maltz, "Dynamic source routing in ad hoc wireless networks," in Mobile Computing, Imielinski and Korth, Eds.Kluwer Academic Publishers, 1996, vol. 353.

[7] C. Perkins and P. Bhagwat, "Highly dynamic destination-sequenced distance-vector routing (DSDV) for mobile computers," in ACM SIGCOMM 94 Conference on Communications Architectures, Protocols and Applications, 1994, pp. 234244.

[8] V. Lenders, M. May, and B. Plattner, "Density-based vs. Proximity-based Anycast Routing for Mobile Networks," in IEEE INFOCOM,Barcelona, Spain, April 2006. 
[9] V. Park and S. Corson, "A Performance Comparison of the TemporallyOrdered Routing Algorithm and Ideal Link-State Routing," Third IEEE Symposium on Computers and Communications, Athens, Greece, 2002.

[10] Rainer Baumann, Simon Heimlicher, Vincent Lenders, Martin May, "Routing Packets into Wireless Mesh Networks," in Proceedings of the IEEE WoWMoM, 2007.

[11] Michael Bahr, "Update on the Hybrid Wireless Mesh Protocol of IEEE 802.11s," IEEE International Conference on Mobile Ad hoc and Sensor Systems, Italy 2008 .

[12] A. Basu, A. Lin, and S. Ramanathan, "Routing Using Potentials: A Dynamic Traffic-Aware Routing Algorithm," in Proceedings of the ACMSIGCOMM03, 2003.

[13] J. Faruque and A. Helmy, "RUGGED: RoUting on finGerprint Gradients in seEnsor Networks," in Proceedings of the IEEE ICPS, 2004.

[14] S. Toumpis and L. Tassiulas, "Packetostatics: Deployement of Massively Dense Sensor Networks as an Electrostatic Problem," in IEEE INFOCOM, 2005.

[15] Q. Fang, J. Gao, and L. J. Guibas, "Locating and bypassing holes in sensor networks," Mobile Networks and Applications, Vol 11, No. 2 pp. 187-200, 2006.

[16] F. Kuhn, R. Wattenhofer, Y. Zhang, and A. Zollinger, "Geometric Ad-Hoc Routing: of Theory and Practice," In twenty-second annual symposium on Principles of distributed computing, pages 6372, 2003.

[17] M. ODell, R. ODell, M. Wattenhofer, and R. Wattenhofer, "Lost in Space Or Positioning in Sensor Networks," In Proceedings of the Workshop on Real-World Wireless Sensor Networks (RE-ALWSN'05), June 2005

[18] Zhou, Anfu, Min Liu, Zhongcheng Li, and Eryk Dutkiewicz. "Joint Traffic Splitting, Rate Control, Routing and Scheduling Algorithm for Maximizing Network Utility in Wireless Mesh Networks." 2015.

[19] Lall, Shruti, B. T. J. Maharaj, and PA Jansen van Vuuren. "Nullfrequency jamming of a proactive routing protocol in wireless mesh networks." Journal of Network and Computer Applications 61 (2016): 133-141.

[20] Ning, Zhaolong, Qingyang Song, Lei Guo, Zhikui Chen, and Abbas Jamalipour. "Integration of scheduling and network coding in multi-rate wireless mesh networks: Optimization models and algorithms." Ad Hoc Networks 36 (2016): 386-397.

[21] Sichitiu, Mihail L. "Wireless mesh networks challenges and opportunities." Electrical and Computer Engineering Department, NC State University, Raleigh, NC, USA (2006).

[22] M. Kozlovszky, A. Balasko , A. Varga, "Enabling OMNeT++-based simulations on grid systems," Proceedings of the 2nd International Conference on Simulation Tools and Techniques, Rome, Italy, 2009. 